\title{
Doppler intra-operatório na cirurgia dos aneurismas cerebrais
}

\author{
Daniel Freire de Figueirêdo*, Djacir Gurgel de Figueirêdo*, Francisco Flávio Leitão de \\ Carvalho*, Francisco Flávio Leitão de Carvalho Filho*, Caio Mendonça**, João Paulo \\ Mattos** $^{* *}$
}

Hospital das Clínicas da Universidade Federal do Ceará

\section{RESUMO}

Os autores apresentam a experiência com o uso do doppler como auxiliar na cirurgia dos aneurismas cerebrais. O método foi usado em 58 pacientes com aneurismas intracranianos, distribuídos da seguinte maneira: 18 aneurismas do complexo comunicante anterior, 14 da artéria cerebral média e 28 da artéria carótida interna (supra e infraclinóidea). Dois pacientes tinham aneurismas múltiplos.

O protocolo utilizado consistiu em testar a patência dos vasos relacionados diretamente com o aneurisma, antes e após a clipagem do mesmo, e testar a total exclusão do aneurisma após a clipagem.

Os autores concluem que o método é extremamente seguro, instantâneo, efetivo, confiável, correlacionando com os resultados angiográficos, reduzindo o tempo e custo do tratamento, diminuindo a necessidade e os riscos da angiografia de controle pós-operatório.

\section{PALAVRAS-CHAVE}

Aneurisma intracraniano. Doppler intra-operatório.

\section{ABSTRACT}

Intraoperative vascular doppler sonography in the surgery of intracranial aneurysms

The authors present their experience in the use of intraoperative vascular doppler sonography in the surgery of intracranial aneurysms. Doppler was used in 58 patients with intracranial aneurysms, distributed as follows: 18 of the anterior comunicating complex, 14 of the sylvian artery and 28 of the carotid artery. Two patients had multiples aneurysms.

The doppler technique was systematically used to evaluate the blood flow in the parent vessel, proximal and distal to the aneurysm before and after the occlusion of the aneurysm and to certify the exclusion of the aneurysm from the circulation.

The authors conclude that the method is efficient for intraoperative assessment of adequate aneurysm clip placement and may substitute postoperative angiography.

\section{KEYWORDS}

Aneurysm. Doppler intraoperative.

\section{Introdução}

Apesar de todos os esforços em busca do sucesso na cirurgia dos aneurismas cerebrais, a maior causa de insucesso está relacionada com a oclusão de vasos ou ramos vasculares próxima à colocação do clipe. A visualização microscópica nem sempre é suficiente para termos certeza da patência dos vasos relacionados com o aneurisma.

\footnotetext{
* Neurocirurgião do Hospital das Clínicas da Universidade Federal do Ceará.

** Estudante de Medicina.
} 
Northes e cols. ${ }^{8,9}$ foram os primeiros a usar o doppler intra-operatório para avaliação hemodinâmica na cirurgia cerebrovascular, em 1979.

Nos últimos dois anos, temos utilizado, nas cirurgias de aneurismas intracranianos, o doppler ultra-sônico de $20 \mathrm{MHz}$ com microprobe de $2 \mathrm{~mm}$ de diâmetro, extremamente preciso. O método é seguro, correlacionando com os resultados angiográficos ${ }^{2}$, poupandose tempo e dinheiro, reduzindo a necessidade de angiografia de controle. Trabalhos estudando pacientes operados de aneurismas cerebrais e malformações arteriovenosas, que são submetidos à angiografia intra ou pós-operatória, mostram a ocorrência de $4 \%$ a $18 \%$ de aneurismas não-clipados ou parcialmente clipados e $0,3 \%$ a $12 \%$ de ramos arteriais incluídos pelo clipe. Danos isquêmicos são, na maioria das vezes, incorrigíveis ${ }^{1,3,7}$.

\section{Material e métodos}

O nosso protocolo consistiu em testar a patência dos vasos relacionados diretamente com o aneurisma, antes e após a clipagem deste, e testar a completa exclusão do aneurisma após a clipagem. Nos últimos casos, temos testado o fluxo no aneurisma antes da clipagem.

Utilizamos o doppler intra-operatório no tratamento de 60 aneurismas de 58 pacientes, todos no sistema carotídeo, sendo 18 na região da artéria comunicante anterior, 14 no território da artéria cerebral média e 28 na artéria carótida intracraniana, tanto em sua porção supra como infraclinóidea. A idade dos pacientes variou de 21 a 71 anos (média de 55). Houve predominância do sexo feminino: 35 mulheres para 23 homens. Em dois casos, os aneurismas eram múltiplos. Todos os pacientes foram operados na graduação de zero a três pontos na escala de Hunt and Hess.

O equipamento utilizado foi um microdoppler cirúrgico da marca Mizuho. Esse aparelho funciona com um sinal de transmissão de $20 \mathrm{MHz}$ de freqüência, com profundidade de penetração de $1 \mathrm{~cm}$. O microprobe, com $2 \mathrm{~mm}$ de diâmetro e em forma de baioneta, permitiu toda a mobilidade e perfeita visão microscópica.

O microprobe deve ser sempre aplicado umedecido com soro fisiológico ou liquor e em angulação de $60^{\circ}$ com o vaso, contra o fluxo sangüíneo.

\section{Resultados}

Nos 60 aneurismas operados, o doppler evidenciou a necessidade de se reposicionar o clipe em 9 (15\%).
Estenose ou oclusão do vaso nutridor, apesar de não existir evidências na visão microscópica, foram os achados mais freqüentes; clipagem de vasos próximos ao aneurisma ocorreu em três (duas vezes com a artéria comunicante posterior e uma vez com a artéria coróidea anterior); presença de fluxo no interior do aneurisma, após a clipagem, ocorreu em 3 casos (5\%).

Não tivemos qualquer tipo de complicação relacionado diretamente com o uso do doppler.

\section{Discussão}

MacDonald e cols. ${ }^{6}$, em uma série de 78 pacientes nos quais realizou angiografia de controle pósoperatório, encontraram clipagem não esperada de vasos relacionados com o aneurisma em $12 \%$ dos casos, 4\% de aneurismas não-clipados e outros 4\% de aneurismas parcialmente clipados (resíduo).

O uso do doppler intra-operatório é um método seguro de auxílio na cirurgia dos aneurismas cerebrais. O procedimento é de fácil uso, com resultado instantâneo, efetivo, confiável e econômico, levando em conta o alto custo dos exames neurorradiológicos. Além de tudo, a angiografia cerebral está associada com uma pequena, mas significante taxa de morbidade. Dos 105 casos de angiografias intra-operatórias descritos por Martin e cols. ${ }^{7}$, ocorreram 3 complicações.

Bailes e cols. ${ }^{2}$ descreveram $100 \%$ de correlação dos dados do uso do doppler intra-operatório com os achados angiográficos intra-operatório e pósoperatório, comprovando a confiabilidade do método.

\section{Referências}

1. ALEXANDER TD, MACDONAL RL, WEIR B: Intraoperative angiography in cerebral aneurysm surgery: a prospective study of 100 craniotomies. Neurosurgery 39:10-8, 1996.

2. BAILES JE, TANTUWAYA LS, FUKUSHIMA T: Intraoperative microvascular doppler sonography in aneurysm surgery. Neurosurgery 40:965-72, 1997.

3. BARROW DL, BOYER KL, JOSEPH GJ: Intraoperative angiography in the management of neurovascular disorders. Neurosurgery 30:153-59, 1992.

4. BOECHER-SCHWARZ HG, UNGERSBOECK K, ULRICH P: Pre and intraoperative methods of controlling cerebral circulation in giant aneurysm surgery. Neurosurg Ver 18:85-93, 1995.

5. GILSBACH JM: Intraoperative Doppler Sonography in Neurosurgery. New York, Springer-Verlag, 1983. 
6. MACDONALD RL, WALLACE MC, KESTLE JRW: Role of angiography following aneurysm surgery. J Neurosurg 79:826-32, 1993.

7. MARTIN NA, BENTSON J, VIÑUELA F, HIESHIMA G, REICHER M, BLACK K, DION J, BECKER D: Intraoperative digital subtraction angiography and the surgical treatment of intracranial aneurysms and vascular malformations. J Neurosurg 73:526-33, 1990.

8. NORNES H, GRIP A, WIKEBY P: Intraoperative evaluation of cerebral hemodynamics using directional doppler technique: 1-Arteriovenous malformations. J Neurosurg 50:145-51, 1979.

9. NORNES H, GRIP A, WIKEBY P: Intraoperative evaluation of cerebral hemodynamics using directional doppler technique: 2-Saccular aneurysms. J Neurosurg 50:570-7, 1979.
10. WROBEL CJ, MELTZER H, LAMOND R, ALKSNE JF: Intraoperative assessment of aneurism clip placement by intravenous fluorescein angiography. Neurosurgery 35:970-3, 1994.

Original recebido em março de 1999

Aceito para publicação em julho de 1999

\section{Endereço para correspondência:}

Daniel Figueirêdo

Av. Beira Mar, 1020/901

CEP 60165-121 - Fortaleza - Ceará

Fone: (0XX85) 219-1202

Fax: (0XX85) 219-3788

E-Mail: dfigueiredo@secrel.com.br 